\title{
Relação entre o estado psicossocial do cuidador informal e o tempo de cuidado dos idosos da região centro de Portugal*
}

\author{
Ricardo Pocinho ${ }^{1}$ \\ (iD) http://orcid.org/0000-0003-1307-5434 \\ Escola Superior de Tecnologia da Saúde de Coimbra, Portugal \\ Pedro Belo ${ }^{2}$ \\ (iD http://orcid.org/0000-0003-1729-9566 \\ Escola Superior de Tecnologia da Saúde de Coimbra, Portugal \\ Cláudia Melo ${ }^{3}$ \\ Escola Superior de Tecnologia da Saúde de Coimbra, Portugal \\ Esperanza Navarro-Pardo \\ (iD http://orcid.org/0000-0002-9355-2909 \\ Universidad de Valencia, España \\ Juan José Fernández Muñoz \\ (iD http://orcid.org/0000-0001-5519-7515 \\ Universidad Rey Juan Carlos, España
}

DOI: http://dx.doi.org/10.17081/eduhum.19.32.2533

Recibido: 20 de junio de 2016

Aceptado: 15 de septiembre de 2016

\section{Relation between informal caregivers and the time spent on elder care in the centre of Portugal}

Palavras-chave:

Cuidador informal, Idoso,

Stress, Apoio social, Demência.

Keywords:

Informal caregiver, Elder,

Stress, Social support, Dementia.

\begin{abstract}
Resumo
O papel desempenhado pela família e cuidadores no tratamento dos idosos é essencial para a promoção e manutenção da sua qualidade de vida, garantindo a sua manutenção do domicílio por um período de tempo mais alargado. Objetivo: Compreender a existência da sobrecarga do cuidador informal e os fatores associados (apoio disponíveis e perfil), bem como estudar o estabelecimento relacional entre o tempo e a sobrecarga. Metodologia: Um questionário de dados sociodemográficos e QASCI (Questionário de Avaliação da Sobrecarga do Cuidador Informal) aplicado a 30 cuidadores da região centro de Portuga. Resultados: São caraterísticas do perfil do cuidador, 45 anos, casado, existência de coabitação e razões relacionadas com o dever e a ligação emocional subjacente.
\end{abstract}

\begin{abstract}
The role played by family and peers, in care for older people is fundamental to the promotion and maintenance of their well-being and their quality of life, ensuring their stay at home as long as possible. Objective: To understand the existence of the informal caregiver problem and associated factors (support, profile). Methodology: for a sample of 30 informal caregivers of seniors was applied and a sociodemographic questionnaire and the QASCI (Evaluationquestionnaire of the Informal Caregiver Overload). Results: The results suggested a caregiver profile characterized by age over 45 years, married, existence of cohabitation and underlying grounds relating to the duty and the emotional connection.
\end{abstract}

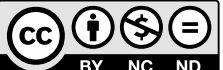

Referencia de este artículo (APA): Pocinho, R., Belo, P., Melo, C., Navarro-Pardo, E. \& Fernández, J. (2017). Relação entre o estado psicossocial do cuidador informal e o tempo de cuidado dos idosos da região centro de Portugal. En Revista Educación y Humanismo, 19(32), 88-101. http://dx.doi.org/10.17081/eduhum.19.32.2533

\footnotetext{
* O artigo referente à linha de pesquisa Envelhecimento e Administração Social, financiado por Coimbra Business School.

1. Escola Superior de Tecnologia da Saúde de Coimbra, Portugal. pocinho@estescoimbra.pt|

2. Escola Superior de Tecnologia da Saúde de Coimbra, Portugal. pedro.belo.santos@gmail.com

3. Escola Superior de Educação de Coimbra, Portugal. claudiarafamello@gmail.com

4. Universidad de Valencia, España. Esperanza.Navarro@uv.es

5. Universidad Rey Juan Carlos, España. juanjose.fernandez@urjc.es
} 


\section{Introdução}

O envelhecimento é um fenómeno transversal a todas as culturas. Contudo, a definição de velhice é algo complexa. A conceção de pessoa idosa deriva, por norma, da fixação de uma idade cronológica que está entre os 60 e os 65 anos (OMS, 1993). Numa outra perspetiva, meramente fisiológica, a velhice é definida como um processo de falência reprodutiva (menopausa/andropausa) causadora de uma progressiva disfunção generalizada, que resulta numa significativa perda da capacidade adaptativa ao ambiente (Coles, 1996). Segundo Weineck (2001), é a soma de todas as alterações biológicas, psicológicas e sociais, que depois de alcançar a idade adulta e ultrapassar a idade de desempenho máximo, levará a uma redução gradual das capacidades de adaptação e do desempenho psicofísico do indivíduo. Na perspetiva de Fraiman (1995), a velhice é um "momento na vida do indivíduo, e um processo extremamente complexo e pouco conhecido, com implicações tanto para quem o vivencia como para a sociedade que o suporta ou assiste a ele" (p.19).

Costa (2002) acrescenta um novo conceito, a senescência, ao dizer que o envelhecimento é um processo segundo o qual o organismo biológico existe no tempo e muda fisicamente, sendo que dele resulta um comprometimento físico e cognitivo, com aumento da vulnerabilidade e probabilidade de morte.

Pode-se ainda dizer que o envelhecimento se traduz na diminuição das capacidades de adaptação ao meio e às agressões de vida. A avaliação da velocidade de processamento é de extrema importância no envelhecimento, pois é um dos primeiros processos a diminuir nos estágios iniciais de demência nesta fase da vida (Faria, Alves \& Charchat-Fichman, 2015).

Segundo Lampert e Rosso (2015) na velhice, a depressão é a doença psiquiátrica mais comum sendo que $23 \%-40 \%$ da população idosa na comunidade tem esse diagnóstico, por outro lado, entre os institucionalizados a taxa de prevalência da mesma é de $54 \%$.

Constata-se, assim, a ausência de uma explicação única para o envelhecimento, visto que este é um processo multifatorial e multidimensional. Designadamente, existem diversas dimensões a considerar quando se procura analisar a pessoa: a biológica, psicológica e sociológica, sendo que todas elas interagem com o meio ambiente (Moniz, 2003).

Do ponto de vista teórico, para Fernández -Ballesteros (2000) são variadas as teorias que procuram explicar o envelhecimento, de acordo com a sua faceta biológica (e.g., teorias genéticas), psicológica (e.g., teorias do desenvolvimento) ou sociológica (e.g., teoria da desvinculação), das quais derivam diversas dimensões do envelhecimento (biológico - alteração progressiva das capacidades; psicológico mudanças ao nível do pensamento e comportamento; sociológico - alteração dos papeis sociais). Salgado (1982) assume uma abordagem 
integradora da velhice, entendendo-a como uma etapa da vida na qual, em consequência da idade cronológica elevada, se verificam modificações de ordem biopsicossocial que afetam a relação entre individuo e meio. Existe uma quebra nas relações familiares e interpessoais, que provoca, igualmente, uma menor qualidade de vida, uma vez que os idosos têm que se familiarizar com o viver sozinhos ou equacionar a possibilidade de perda de independência. Deste modo, não só não se sentem úteis no âmbito familiar, como muitas das vezes é necessário que alguém cuide deles devido problemas de saúde derivados da idade. O processo de envelhecimento surge acompanhado de um declínio leve no funcionamento cognitivo e uma queixa subjetiva de memória torna-se frequente na população idosa, podendo estar associada a mudanças no humor e/ou cognitivas e que incrementa a possibilidade de demência (Brigola et al., 2015).

Com o aumento da qualidade de vida, da melhoria das condições de vida, aumento da esperança média de vida, a par com a taxa de envelhecimento, colocaram a sociedade perante uma população maioritariamente envelhecida. Vários fatores têm vindo a configurar o cenário que carateriza atualmente a terceira idade; a estrutura familiar alargada que ilustrava o nosso país há algumas décadas atrás (e que consistia na coabitação de várias gerações que cuidavam umas das outras, assegurando uma velhice com um mínimo de suporte) deu lugar a um núcleo familiar restrito; a entrada generalizada da mulher no mundo do trabalho; o ritmo de vida frenético dos nossos dias; o tipo de habitação em prédios; a vida impessoal das cidades, entre outros motivos, vieram acentuar uma série de circunstâncias de carência em que muitos idosos se encontram, implicando a necessidade de se encontrar respostas para esta faixa da população, na qual, ainda que deva ser contrariada uma imagem de velhice negativa, é inegável a existência de um conjunto de problemáticas, particularmente associadas à saúde, a que é necessário atender. Associadas ao funcionamento cognitivo na velhice, sendo que a memória é a função cognitiva mais afetada, surge a fraqueza muscular e a perda de mobilidade. $\mathrm{O}$ comprometimento cognitivo é mais prevalente nos idosos frágeis, e quanto maior a fragilidade, maior o risco de demência. A presença concomitante de fragilidade e mudanças cognitivas podem auxiliar a prever a mortalidade nos idosos (Brigola et al., 2015).

Ainda que as respostas para esta população surjam exteriores à família, esta continua a deter um papel fulcral no cuidado ao idoso. Se numa primeira fase, o apoio ao idoso se centra na rede social próxima, constituída por familiares, amigos e vizinhos, um conjunto de constrangimentos pessoais, históricos, sociais e culturais, alteraram a forma de perspetivar a terceira idade ao longo do tempo, alterando conceções, necessidades e respostas, fazendo surgir as instituições direcionadas para o acolhimento de idosos (Guimarães, 1999), que frequentemente coexistem com os cuidadores no apoio à terceira idade. Ainda que o poio social tome 
um papel de destaque na sociedade atual, quer por motivos financeiros, seja por questões de vínculo familiar, muitos idosos encontram-se ao cuidado de alguém ou em situações mistas.

Esta é uma atividade complexa, quer pela sua dimensão psicológica quer pela dimensão social, ética e demográfica, albergando vários aspetos de ordem clínica, técnica e comunitária. Cuidar de alguém passa por garantir o seu bem-estar, a sua saúde, alimentação, higiene pessoal, e aspetos como a recreação e lazer (Garcia, 2013). Cuidar implica a responsabilidade de tomar conta de alguém, satisfazer/responder às suas necessidades e sentir preocupação, interesse, consideração e afeto pela pessoa de quem se cuida (Sequeira, 2010). Braithwaite (2000, citado por Martins, Ribeiro \& Garrett, 2003) define Cuidador Informal (CI) como "a pessoa não remunerada, familiar ou amiga que se assuma como principal responsável pela organização ou assistência e prestação de cuidados à pessoa dependente" (p.137).

Importa distinguir cuidadores principais aqueles que têm a seu cargo toda ou grande parte da responsabilidade pelos cuidados à pessoa idosa no domicílio, realizando todas as tarefas que este não consiga desenvolver, de cuidadores secundários - vizinhos, voluntários e/ou mesmo alguns familiares que prestam cuidados de uma forma parcial, e esporádica. De modo semelhante, existem cuidadores formais e cuidadores informais, sendo os primeiros profissionais pagos pelos seus cuidados prestados à pessoa idosa e normalmente possui formação para tal, e os segundos familiares, vizinhos, amigos que prestam cuidados à pessoa idosa sem serem remunerados por tal atividade (Sequeira, 2010).

Normalmente o ato de cuidar recai sobre um único membro da família, o cuidador primário, associado na maior parte das vezes ao cônjuge oua descendência direta; estes são, maioritariamente, mulheres (Marques, 2000), numa lógica cultural em que é a mulher que assume um papel ativo na prestação de cuidados, a par com a realização de tarefas domésticas (Nakatano, Souto, Paulette, Melo \& Sousa, 2003); a coabitação está diretamente associada ao estado civil do prestador de cuidados pois o dividir a habitação com a pessoa idosa incapacitada expõe o cuidador à realidade do idoso, levando-o a tornar-se cuidador; quanto aos motivos que levam ao cuidador informal, são apontados a preocupação em evitar a institucionalização, constituindo um último recurso (Jani-le-Bris, 1994); em termos profissionais, são maioritariamente domésticos e reformados da sua antiga profissão. Figueiredo (2007) refere que os motivos subjacentes à assunção do papel de cuidador a um idoso dependente constituem um domínio complexo que envolve uma miscelânea razões, marcado pelas tradições, pelos padrões e normas sociais vigentes em cada cultura, pela conceção de vida e história de cada indivíduo.

Cuidar apresenta duas componentes principais: trabalho emocional, que envolve a relação dual entre o idoso e o cuidador e trabalho de gestão e de organização das atividades diárias que o idoso deverá realizar. Tomar conta de um 
parente idoso dependente pressupõe o envolvimento emocional, esforço físico e dispêndio de tempo e energia, que dificilmente podem ser entendidos como algo insignificante na vida dos indivíduos, acarretando, habitualmente, custos elevados para a saúde e para o bem-estar do cuidador. $\mathrm{O}$ impato pode acontecer na esfera objetiva - familiar, social, económica e profissional, ou subjetiva - respostas emocionais (Figueiredo, 2007).

Para Maslach, Schaufeli e Leiter (2001) o burnout, ou esgotamento, poderá manifestar-se em exaustão física, emocional e despersonalização. Benevides-Pereira (2002) refere que os sintomas desta síndrome podem ser divididos em físicos/emocionais (sensação de fadiga constante, perturbação do sono, etc.) ou comportamentais (Irritabilidade, incapacidade de relaxar, aumento do consumo de bebidas alcoólicas, suicídio).

\section{Problema de Investigação}

O objetivo desde trabalho pretendeu analisar a relação existente entre o cuidador informal e o tempo de suporte social despendido nos cuidados com a população idosa.

O grau de exigência dos cuidados influencia o quotidiano do cuidador, alterando, frequentemente a sua qualidade de vida. Esta noção surge, para a Organização Mundial de Saúde (1993), como a perceção do indivíduo de sua posição na vida, no contexto cultural e de sistemas de valores no qual vive e em relação às suas metas, expetativas, valores e preocupações. A sobrecarga constitui uma resposta psicossocial, uma consequência que inclui a diminuição de sentimentos de bem-estar, stresse e um aumento dos problemas de saúde (Pearlin, Mullan, Semple \& Skaff, 1990; Figueiredo, 2007; Vitaliano, Scanlan \& Zhang, 2003). A qualidade de vida do cuidador assume contornos positivos ou negativos, consoante as estratégias adaptativas que põe em prática e os recursos que mobiliza e ativa, face aos constrangimentos de cuidar de alguém dependente. A investigação que tem vindo a ser desenvolvida acerca dos cuidadores informais tem dado especial atenção às consequências negativas que advêm da experiência de cuidar (Jenaro, Grueso, Flores, Navarro-Pardo \& Vázquez-Martínez, 2013). No entanto, este fato dá uma imagem muito redutora desta experiência, pois exclui os impatos positivos; a prestação de cuidados não pode ser vista apenas como uma fonte stressante, mas também como uma fonte de gratificação e realização pessoal. $\mathrm{Na}$ experiência de cuidar existem "aspetos potencialmente satisfatórios e gratificantes da situação de prestar cuidados a um familiar" (Brito, 2002, p.45). As recompensas ou as experiências positivas na experiência do cuidar incluem um sentido de satisfação, de gratificação e de orgulho no papel de cuidar, aumento do sentido de domínio, competência e realização, bem como um sentido de finalidade e significado para a vida (Dupuis, Epp \& Smale, 2004). Estes autores acrescentam ainda os benefícios emocionais, o sentimento de reciprocidade, o crescimento e desenvolvimento pessoal e o aumento da qualidade no relacionamento social, (sobretudo, com o recetor de cuidados) como aspetos gratificantes que derivam da experiência de cuidar. 
Em termos formais, existem três tipos de programas de suporte direcionados aos cuidadores: grupos de apoio; grupos de aprendizagem conduzidos por profissionais; grupos de aconselhamento/acolhimento, que, atendendo às necessidades de cada idoso/cuidador, permitem reduzir o isolamento, aumentar conhecimentos sobre o processo de envelhecimento e os problemas de saúde específicos para cada situação, melhorar o desempenho do cuidador através de habilidades e estratégias, visando o bem-estar físico, emocional do cuidador e a redução da sua sobrecarga, melhorando a sua Qualidade de Vida.

\section{Metodologia}

A amostra foi constituída por 30 Cuidadores Informais (CI) de idosos da zona centro de Portugal (Coimbra e Pombal). Fizeram parte da amostra de estudo apenas os profissionais que concordaram com o Termo de Consentimento apresentado, o qual assegurava a questão de anonimato e confidencialidade.

Os instrumentos aplicados foram um Questionário Sociodemográfico e o QASCI - Questionário de Avaliação da Sobrecarga do Cuidador Informal (Martins, Ribeiro \& Garrett, 2003). Este é composto por 32 itens, avaliados numa escala do tipo Likert. Inclui 7 dimensões: Implicações na Vida Pessoal do cuidador (11 itens); Satisfação com o Papel e com o Familiar (5 itens); Reacções a Exigências (5 itens); Sobrecarga Emocional relativa ao familiar (4 itens); Suporte Familiar (2 itens); Sobrecarga Financeira (2 itens) e a Perceção de Mecanismos de
Eficácia e de Controlo (3 itens). Em três subescalas (Satisfação com o Papel e com o Familiar, Suporte Familiar e Perceção de Mecanismos de Eficácia e de Controlo), as pontuações são invertidas (Martins, Ribeiro \& Garrett, 2003).

A subescala Implicações na Vida Pessoal pretende avaliar as repercussões sentidas pelo cuidador por estar a cuidar do seu familiar, como seja a diminuição de tempo disponível para si, sentir a sua saúde e a sua vida social serem afetadas, sentir um grande esforço físico por estar a tomar conta da pessoa dependente e deparar-se com planos alterados na sua vida, bem como, a sensação de estar preso (Martins, Ribeiro \& Garrett, 2003). A subescala Satisfação com o Papel e com o Familiar diz respeito a sentimentos e emoções positivas, aumento de auto-estima e sentir-se mais próximo do doente, decorrentes do desempenho do papel e da relação afetiva que o cuidador estabelece com o sujeito alvo dos cuidados (Martins, Ribeiro \& Garrett, 2003). A subescala Reacções a Exigências inclui sentimentos negativos, como a perceção de manipulação por parte do doente, ou a presença de comportamentos por parte deste capaz de levar a embaraço do cuidador, sentimentos de ofensa e diminuição da privacidade. A Sobrecarga Emocional compreende emoções negativas do cuidador, que podem levar a conflitos internos e sentimentos de fuga, cansaço e esgotamento perante a situação em que se encontram (Martins, Ribeiro \& Garrett, 2003). O Suporte Familiar diz respeito ao reconhecimento e apoio que a família disponibiliza ao cuidador. À subescala Sobre- 
carga Financeira estão inerentes as dificuldades económicas decorrentes da situação de doença do familiar e à incerteza do futuro económico. A subescala Perceção de Mecanismos de Eficácia e de Controlo compreende aspetos que capacitam ou facilitam ao cuidador continuar a enfrentar os problemas que advêm do desempenho de papel, como sejam, a capacidade para tomar conta do doente dependente e os conhecimentos e experiência para assumir a responsabilidade do cuidado (Martins, Ribeiro \& Garrett, 2003).

\section{Resultados}

O questionário mostou boa consistência interna, apresentando um alfa de Cronbach de 0.90 .

De seguida será feita a análise descritiva dos dados sociodemográficos, os quais estão presentes na Tabela 1.

No que toca à idade, verifica-se que a totalidade da amostra se insere numa faixa etária acima dos 40 anos (46-83), sendo que $60 \%$ tem menos de 60 anos. A maioria dos cuidadores informais é do sexo feminino (N=27;90\%) e $60 \%$ $(\mathrm{N}=18)$ estão casados. $\mathrm{Na}$ amostra estudada, $16,7 \%(\mathrm{~N}=5)$ sujeitos são solteiros, enquanto $23,3 \%$ da amostra é cuidador há menos de 2 anos $(\mathrm{N}=7)$ e $30 \%(\mathrm{~N}=9)$ exerce essa atividade entre 2 e 5 anos. Na amostra 7 cuidadores (23,3\%) cuida entre 5 e 10 anos de alguém e a mesma percentagem foi encontrada com funções de cuidados há mais de 10 anos. Verificamos que 17 sujeitos $(56,7 \%)$ são o único cuidador da pessoa, enquanto $13(43,3 \%)$ tem apoio de outrem.
Tabela 1. Perfil dos cuidadores informais $(\mathbf{N}=\mathbf{3 0})$

\begin{tabular}{|c|c|c|c|}
\hline & & $\mathbf{N}$ & $\%$ \\
\hline \multirow{2}{*}{ Idade } & $<40$ anos & 0 & $0 \%$ \\
\hline & $>40$ anos & 30 & $100 \%$ \\
\hline \multirow{2}{*}{ Género } & Feminino & 27 & $90 \%$ \\
\hline & Masculino & 3 & $10 \%$ \\
\hline \multirow{6}{*}{ Profissão } & $\begin{array}{l}\text { Espec. intelectuais } \\
\text { científicos }\end{array}$ & 4 & $13,3 \%$ \\
\hline & Técn. nível intermedio & 4 & $13,3 \%$ \\
\hline & Adm. e similar & 3 & $10 \%$ \\
\hline & Serv. e vendedores & 6 & $20 \%$ \\
\hline & Não qualificados & 3 & $10 \%$ \\
\hline & Reformados & 10 & $33 \%$ \\
\hline \multirow{4}{*}{ Estado civil } & Solteiro & 5 & $16,7 \%$ \\
\hline & Casado & 18 & $60 \%$ \\
\hline & Divorciado & 4 & $13,3 \%$ \\
\hline & Viúvo & 3 & $10 \%$ \\
\hline \multirow{5}{*}{ Parentesco } & Cônjuge & 6 & $20 \%$ \\
\hline & Filha/filho & 18 & $60 \%$ \\
\hline & Nora & 2 & $6,7 \%$ \\
\hline & Irmã/irmão & 2 & $6,7 \%$ \\
\hline & Não tem & 2 & $6,7 \%$ \\
\hline \multirow{2}{*}{ Coabitação } & Sim & 21 & $70 \%$ \\
\hline & Não & 9 & $30 \%$ \\
\hline \multirow{4}{*}{ Tempo } & $<2$ anos & 7 & $23,3 \%$ \\
\hline & $2-5$ anos & 9 & $30 \%$ \\
\hline & 5-10 anos & 7 & $23,3 \%$ \\
\hline & $>10$ anos & 7 & $23,3 \%$ \\
\hline \multirow{2}{*}{ Único cuidador } & Sim & 17 & $56,7 \%$ \\
\hline & Não & 13 & $43,3 \%$ \\
\hline \multirow{6}{*}{ Motivos } & Dever-Dependência & 8 & $26,7 \%$ \\
\hline & Dever-Parentesco & 15 & $50 \%$ \\
\hline & Características instituições & 1 & $3,3 \%$ \\
\hline & Valorização-lar & 2 & $6,7 \%$ \\
\hline & Financeiros & 1 & $3,3 \%$ \\
\hline & Profissionais & 1 & $3,3 \%$ \\
\hline
\end{tabular}

Relativamente à profissão, $33 \%$ dos sujeitos $(\mathrm{N}=10)$ estão reformados; verificaram-se ainda 4 $(13,3 \%)$ especialistas nas profissões intelectuais e científicas, assim como técnicos e profissionais de nível intermédio. 
Atendendo ao grau de parentesco, 18 sujeitos (60 \%) são filhos da pessoa cuidada e $6(20 \%)$ são o cônjuge. Assim, sendo a maioria dos cuidadores mulheres, e a maioria dos cuidadores são filhos das pessoas cuidadas, isso significa que a tarefa social de cuidar do idoso é geralmente atribuída às filhas dos idosos.

No que diz respeito à continuidade do cuidado, existem na amostra 20 sujeitos (66,7 $\%)$ que prestam os cuidados em continuidade. O motivo principal para prestar este cuidado prendese com o grau de parentesco para com o idoso $(\mathrm{N}=15)$.

Quanto à análise dos apoios disponibilizados aos cuidadores informais, foram encontrados os seguintes resultados, indicados na Tabela 2.

Tabela 2. Apoios disponibilizados aos Cuidadores Informais $(\mathbf{N}=\mathbf{3 0})$

\begin{tabular}{|c|c|c|c|}
\hline & & $\mathbf{N}$ & $\%$ \\
\hline \multirow{2}{*}{$\begin{array}{l}\text { Acesso Informação } \\
\text { patologia }\end{array}$} & Sim & 13 & $43,3 \%$ \\
\hline & Não & 17 & $56,7 \%$ \\
\hline \multirow{4}{*}{ Onde informação } & Instituições Apoio & 13 & $43,3 \%$ \\
\hline & Hospital/Médicos & 9 & $30 \%$ \\
\hline & Formação Profissional & 2 & $6,7 \%$ \\
\hline & Outros & 2 & $6,7 \%$ \\
\hline \multirow{2}{*}{$\begin{array}{l}\text { Participação } \\
\text { Formação patologia }\end{array}$} & Sim & 6 & $20 \%$ \\
\hline & Não & 24 & $80 \%$ \\
\hline \multirow{2}{*}{$\begin{array}{l}\text { Participação } \\
\text { Formação cuidador }\end{array}$} & Sim & 4 & $13,3 \%$ \\
\hline & Não & 26 & $86,7 \%$ \\
\hline \multirow{2}{*}{$\begin{array}{l}\text { Informação } \\
\text { Associações apoio }\end{array}$} & Sim & 10 & $33,3 \%$ \\
\hline & Não & 20 & $66,7 \%$ \\
\hline \multirow{2}{*}{ Grupo partilha } & Sim & 6 & $20 \%$ \\
\hline & Não & 24 & $80 \%$ \\
\hline \multirow{2}{*}{$\begin{array}{l}\text { Contacto } \\
\text { Profissionais }\end{array}$} & Sim & 23 & $76,7 \%$ \\
\hline & Não & 7 & $23,3 \%$ \\
\hline \multirow[b]{2}{*}{ Onde } & Hospital/centro saúde & 13 & $43,3 \%$ \\
\hline & $\begin{array}{l}\text { Profissionais de } \\
\text { associações }\end{array}$ & 9 & $30 \%$ \\
\hline
\end{tabular}

\begin{tabular}{|l|c|c|c|}
\hline \multirow{2}{*}{ Adaptação espaços } & Esporadicamente médico & 2 & $6,7 \%$ \\
\cline { 2 - 4 } & Profissionalmente & 2 & $6,7 \%$ \\
\cline { 2 - 4 } & Sim & 10 & $33,3 \%$ \\
\hline \multirow{2}{*}{ Apoio económico } & Não & 20 & $66,7 \%$ \\
\cline { 2 - 4 } & Sim & 3 & $10 \%$ \\
\hline \multirow{2}{*}{ Apoio Jurídico } & Não & 27 & $90 \%$ \\
\cline { 2 - 4 } & Não & 29 & $96,7 \%$ \\
\hline \multirow{2}{*}{ Outro apoio } & Sim & 1 & $3,3 \%$ \\
\cline { 2 - 4 } & Sim & 7 & $23,3 \%$ \\
\hline
\end{tabular}

Da amostra analisada, 13 sujeitos (43,3\%) obtiveram informação sobre a patologia da pessoa de quem cuidam, nos seguintes locais: instituições de apoio ( $\mathrm{N}=13 ; 43,3 \%)$, hospital/ médicos $(\mathrm{N}=9 ; 30 \%)$. A amostra apresenta $80 \%$ dos cuidadores $(\mathrm{N}=24)$ que não tiveram formação sobre a patologia da pessoa a seu cuidado.

Relativamente à formação do cuidador, apenas 4 sujeitos $(13,3 \%)$ tiveram nesse grupo de apoio. Quanto ao conhecimento de programas no âmbito da patologia da pessoa cuidada, apenas 10 sujeitos (33,3 \%) tiveram informação, todos eles em associações relacionadas (e.g., centro de dia). Relativamente ao conhecimento de associações de apoio, 66,7 \% (N=20) não tiveram informação sobre este tipo de instituições. Apenas 6 sujeitos (20\%) integram grupos de partilha, ainda que a maioria $(\mathrm{N}=23$; $76,7 \%$ ) contacte com profissionais que podem esclarecer dúvidas eventuais.

Para exercer o cuidado à pessoa a seu cargo, apenas 33,3 \% $(\mathrm{N}=10)$ da amostra precisou de fazer alterações na sua residência, sendo sobretudo no quarto da pessoa cuidada. 
Procurou-se ainda perceber a disponibilidade dos sujeitos para participar em ações informativas e de formação através da importância a elas atribuída; $90 \%$ da amostra ( $\mathrm{N}=27)$ considerou ser "muito importante" ter acesso a informação sobre a patologia da pessoa de quem cuidam; 25 sujeitos $(83,3 \%)$ consideraram "muito importante" participar em formações nesse âmbito; 24 sujeitos (80 \%) tiveram a mesma opinião tratando-se de formações para o cuidador e 25 sujeitos $(83,3$ \%) sobre ter informação acerca de associações vocacionadas para essa questão. $\mathrm{Na}$ amostra analisada $63,3 \%$ dos sujeitos $(\mathrm{N}=19)$ responderam ser "muito importante" a participação em grupos de partilha e 25 cuidadores $(83,3 \%)$ pensam o mesmo sobre o contato com profissionais de referência.

Para a avaliação da sobrecarga do cuidador os resultados estão indicados na Tabela 3.

Tabela 3. Média e desvio-padrão das respostas dadas pelos cuidadores informais na escala QASCI e suas dimensões $(\mathbf{N}=30)$

\begin{tabular}{|l|c|c|}
\hline & Média & DP \\
\hline Sobrecarga Emocional relativa ao familiar & 27,92 & 1,44 \\
\hline $\begin{array}{l}\text { Implicações na Vida Pessoal do familiar } \\
\text { cuidador }\end{array}$ & 48,56 & 0,04 \\
\hline Sobrecarga Financeira & 31,67 & 1,08 \\
\hline Reações às Exigências & 60,00 & 8,40 \\
\hline $\begin{array}{l}\text { Perceção dos Mecanismos de Eficácia e } \\
\text { Controlo }\end{array}$ & 35,56 & 2,89 \\
\hline Suporte Familiar & 27,92 & 7,23 \\
\hline Satisfação com o Papel e com o Familiar & 21,27 & 7,23 \\
\hline QASCI Total & 37,7 & 2,13 \\
\hline
\end{tabular}

Ainda que não existam pontos de corte estabelecidos, é sugerida a seguinte (Loureiro et al., 2013) análise normativa: " $0=$ Ausência de Sobrecarga"; "1-25 = Sobrecarga Ligeira"; "25-50 = Sobrecarga Moderada"; "50-75 = Sobrecarga Grave"; e ">75 Sobrecarga Extremamente Grave".

Assim, considerando a escala global, a média de pontuação obtida foi de $37.7(\mathrm{dp}=2.13)$, correspondendo a uma sobregarga moderada. Atendendo a uma perspetiva mais específica, por subscala, apenas na satisfação com o papel e com o familiar a amostra apresenta uma sobrecarga ligeira (média $=21.27 ; \mathrm{dp}=7.23$ ); na reação às exigências, os sujeitos da amostra surgem como tendo uma sobrecarga grave (média $=60.00$; $\mathrm{dp}=8.4$ ), sugerindo a presença de sentimentos negativos, como a perceção de manipulação por parte do doente, sentimentos de ofensa e diminuição da privacidade; nas restantes substacalas, a amostra apresenta uma sobrecarga moderada.

Foi estudada a relação existente entre a sobrecarga do cuidador informal e o tempo de prestação de cuidado através do $r$ de Pearson, estando os resultados indicados na Tabela 4.

Tabela 4. Correlação entre a QASCI e suas dimensões e características do cuidado/apoios existentes

\begin{tabular}{l|l|l|l|l|l|l|l|l|}
\hline & SE & IVP & SF & RE & PMEC & SFM & SPF & QASCI \\
\hline TC &,- 015 &,- 046 &,- 065 &, 089 &, 287 &, 146 &, $382 *$ &, 093 \\
\hline
\end{tabular}

O tempo de prestação de cuidado correlacionou-se de forma significativa e positiva com o papel e satisfação com o familiar $(r=.382 ; \mathrm{p}<.05)$. 


\section{Discussão}

$\mathrm{O}$ fato dos cuidadores o serem a tempo inteiro surge como fator de satisfação com esse papel para com a pessoa cuidada. O tempo de prestação de cuidado correlacionou-se, neste estudo, de forma positiva, com o papel e satisfação com o familiar, sugerindo a presença de emoções positivas decorrentes da ligação "pessoa cuidada" - "cuidador". Tal, surge reforçado quando verificamos que os motivos que levam estes cuidadores a desempenhar este papel se prende fortemente com a ligação emocional já existente. O estudo de Dávila e Morejón (2012) permite perceber que é a ligação emocional que fornece uma estrutura propícia para a compreensão do mundo afetivo dos idosos.

Por outro lado, um maior apoio social pode lançar as bases para uma melhor perceção de saúde, o que em conjunto diminuiu o stress, prevenindo o desenvolvimento de depressão entre os cuidadores (Junior, Blumentahl \& Garner, 2015).

O stress crônico experimentado pelos cuidadores, está associado a um humor negativo (Leggett, Zarit, Kim, Almeida \& Klein, 2015), pelo que o fato de surgir um ponto de satisfação pessoal pode eliminar este tipo de humor, tal como foi encontrado nos nossos resultados.

De acordo com o estudo de Williams, Zimmerman (2011), mais de metade dos cuidadores familiares fazem a monitorização, gestão e assistência com as refeições e 40 \% auxiliam nas tarefas de cuidados pessoais. Quando este envolvimento se relaciona com uma necessidade percebida, não se encontrando na amostra humor negativo, nem depressão.

Ainda assim, a amostra estudada apresentou, na generalidade, uma sobrecarga moderada, destacando-se com maior impacto a reação às exigências, ou seja, a a presença de sentimentos negativos, como a perceção de manipulação por parte do doente, sentimentos de ofensa e diminuição da privacidade. Neste sentido, a existência de programas comunitários de educação e suporte destinados aos cuidadores, constituem um importante recurso na otimização das ferramentas ao dispor do cuidador, e na manutenção da sua própria saúde e bem-estar. Esta ideia parece suportada pelos nossos resultados, $80 \%$ dos sujeitos consideraram muito importante a existência destes tipos de apoio.

Os cuidadores com mais tempo de serviço apresentam níveis mais baixos de rigidez e estão melhor informados sobre os serviços, passando a compartilhar responsabilidades de cuidar, o que vem ao encontro da satisfação pessoal (Herrera, Lee, Palos \& Torres-Vigil, 2008).

\section{Conclusão}

Ainda que as respostas sociais para a terceira idade tenham vindo a aumentar, existem idosos que permanecem junto de familiares, ou outras pessoas significativas que assumem o papel de cuidadores. Num momento mais avançado, o idoso poderá depender integralmente de um 
cuidador, frequentemente parente ou pessoa próxima.

A nível pessoal, os cuidadores ficam sem tempo para si próprios, para os outros que lhe estão próximos e para o desenvolvimento de atividades sociais e culturais; alguns cuidadores mantêm a sua atividade laboral, sem qualquer tipo de restrição ou adaptação, mas outros há que têm de abdicar da mesma; numa situação intermédia, têm de fazer alguns ajustamentos mas conseguem conciliar os cuidados e a vida profissional (Sequeira, 2010; Isaac, Stewart \& Krishnamoorthy, 2010). O grau de dependência dos idosos é ligeiro ou porque seja possível recorrer a serviços formais ou informais complementares; ao nível financeiro, algumas situações implicam um dispêndio elevado de dinheiro, habitualmente suportado pelo próprio idoso; o facto de os cuidadores necessitarem de responder às exigências colocadas pelos idosos e às solicitações dos outros familiares tem implicações relacionais, podendo a própria relação com o idoso que é cuidado ser ambígua e potenciadora de situações de stress (Sequeira, 2010; Isaac, Stewart \& Krishnamoorthy, 2010). Estas ideias apontam a necessidade de estudar o impacto no cuidador desta atividade e de lhe garantir mecanismos capazes de atenuar estas repercussões, que ao influenciarem a sua qualidade de vida, alcançam, também, a pessoa cuidada.

Explana-se a importância da família no cuidado à pessoa dependente, visto como um dever relacionado com a afetividade. No entanto, as respostas dos cuidadores parecem sugerir que as ofertas exteriores ao domicílio não serão, também, as mais adequadas e possíveis de comportar, carecendo da componente emocional, quer implicando custos superiores ao possível para a maioria das pessoas. De facto, nem sempre os mecanismos disponíveis, quando os há, são os mais adequados, ou estão ao alcance de todos, tal como refere Iecovich (2008).

No entanto, os familiares cuidadores de idosos com demência, ainda que sentindo satisfação por este papel, estão sujeitos a inúmeras fontes de stress, decorrente da assunção de um papel para qual, frequentemente, não estão preparados, assim como das repercussões na sua vida diária (Ramos, 2004).

A perceção de que a população idosa existe, cada dia que passa em maior número, torna urgente que se considerem recursos para esta faixa da população, nomeadamente no que respeita à restruturação das políticas sociais, sendo que a promoção da autonomia e independência na pessoa idosa são aspetos fundamentais para o individuo e, consequentemente, para as suas redes sociais próximas e sociedade onde se insere.

\section{Bibliografia}

Benevides-Pereira, A.M.T. (2002). O processo de adoecer pelo trabalho. In A.M.T., Benevides-Pereira (org.). Burnout: quando o trabalho ameaça o bem-estar do trabalhador. São Paulo: Casa do Psicólogo. 
Brigola, A.G., Manzini, C.S.S., Oliveira, G.B.S., Ottaviani, A.C., Sako, M.P., \& Vale, F.A.C. (2015). Subjective memory complaints associated with depression and cognitive impairment in the elderly: a systematic review. Dementia \& Neuropsychology, 9(1), 51-57.

Brigola, A.G., Rossetti, E.S., Santos, B.R., Neri, A.L., Zazzetta, M.S., Inouye, K., \& Pavarini, S. (2015). Relationship between cognition and frailty in elderly: A systematic review. Dementia \& Neuropsychology, 9(2), 110-119.

Brito, L. (2002). A Saúde mental dos prestadores de cuidados familiares idosos. Coimbra: Quarteto Editora.

Coles, L. S. (1996). Theories of Aging: the fable of the blind men touching the elephant. Recuperado no 22 de abril de 2015 em: http://home.esrthlink.net/ scoles/

Costa, M. A. (2002). Cuidar idosos: Formação, prática e competências dos enfermeiros. Coimbra: Formasau.

Dávila, Z., \& Morejón, S. (2012). Un estudio exploratorio de capacidades emocionales en adultos mayores. Psicogente, 15(27), 49-61.

Dupuis, S.L., Epp, T., \& Smale, B.J.A. (2004). Caregivers of persons with dementia: Roles, experiences, supports, and coping. Literature review prepared for the Ministry of Health and Long-Term Care and the Ontario Senior's Secretariat as part of Ontario's Alzheimer Stratety (Initiative \#6 Research on Caregiver
Needs). Waterloo: Murray Alzheimer Research and Education Program.

Faria, C.A., Alves, H.V.D., \& Charchat-Fichman, H. (2015). The most frequently used tests for assessing executive functions in aging. Dementia \& Neuropsychology, 9(2), 149-155.

Fernández-Ballesteros, R. (2000). Gerontología social. Madrid: Ediciones Pirámide.

Figueiredo, L. (2007). Cuidados familiares ao idoso dependente. Lisboa: Climepsi Editores.

Fontán, L. (2012). La Enfermedad de Alzheimer: elementos para el diagnóstico y manejo clínico en el consultorio. Biomedecina, 7(1), 34 - 43. ISSN 1510-9747

Fraiman, A. (1995). Coisas da idade. São Paulo: Gente.

Garcia, E. (2013). Territorialidad y reivindicación social: reflexiones en torno al barrio de La Prosperitat/Barcelona desde la perspectiva del adulto mayor. Revista Educación y Humanismo, 25(15), 59-72.

Guimarães, P. (1999). Os direitos dos idosos. In Atas do Seminário "Envelhecer: um direito em construção”, Lisboa, 1-2 Fev. 1999.

Herrera, A., Lee, J., Palos, G., \& Torres-Vigil, I. (2008). Cultural Influences in the Patterns of Long-Term Care Use Among Mexican American Family Caregivers. Journal of Applied Gerontology, 27(2), 141-165. doi: $10.1177 / 0733464807310682$

Iecovich, E. (2008). Caregiving Burden, Community Services, and Quality of 
Life of Primary Caregivers of Frail Elderly Persons. Journal of Applied Gerontology, 27(3), 309-330. doi: $10.1177 / 0733464808315289$

Isaac, V., Stewart, R., \& Krishnamoorthy, E. (2011). Caregiver Burden and Quality of Life of Older Persons With Stroke - A Community Hospital Study in South India. Journal of Applied Gerontology, 30(5), 643-654. doi: $10.1177 / 0733464810369340$

Jani-le-Bris, H. (1994). Responsabilidade Familiar pelos Dependentes Idosos nos Países das Comunidades Europeias. Irlanda: Fundação Europeia para a Melhoria das Condições de Vida e de Trabalho.

Jenaro, C., Grueso, A., Flores, N. Navarro-Pardo, E., \& Vázquez-Martínez, A. (2013). La experiencia de cuidar en primera persona: Vivencias de cuidadores informales de enfermos de Alzheimer. Revista Ibero Americana de Gerontologia, 1, 10-26.

Junior, B. H., Blumenthal, H., \& Garner, A. (2015). Social Support and Grandparent Caregiver Health: One-Year Longitudinal Findings for Grandparents Raising Their Grandchildren. Journal of Gerontology, Series B Psychol Sci Soc Sci, 70(5), p. 804-812. doi: 10.1093/geronb/gbu165

Lampert, M.A., \& Rosso, A.L.P. (2015).

Depression in elderly women resident in a long-stay nursing home. Dementia \& Neuropsychology, 9(1), 76-80.

Leggett, A., Zarit, S., Kim, K., Almeida, D., \&
Klein, L. C. (2015). Depressive Mood, Anger, and Daily Cortisol of Caregivers on High- and Low-Stress Days. J Gerontol B Psychol Sci Soc Sci, 70(6), 820-829. doi: 10.1093/geronb/gbu070

Marques, S. (2000). Cuidadores familiares de idosos: relatos de histórias. Dissertação de Mestrado, Escola de Enfermagem - Universidade de São Paulo, Ribeirão Preto, Brasil.

Martins, T. (2006). Acidente Vascular Cerebral: Qualidade de Vida e Bem-Estar dos Doentes e Familiares Cuidadores. Coimbra: Formasau.

Martins, T., Ribeiro, J., \& Garrett, C. (2003). Estudo de validação do questionário de avaliação da sobrecarga para cuidadores informais. Psicologia, Saúde \& Doenças, 4(1), 131-148. ISSN: 1645-0086

Martins, T., Ribeiro, J., \& Garrett, C. (2004). Questionário de Avaliação do Cuidador Informal (QASCI) - Reavaliação das Propiedades Psicométricas. Referência, 11, 17-31.

Maslach, C., Schaufeli, W. B., \& Leiter, M. P. (2001). Job Burnout. Annua. Review of Psychology, 52, 397-422.

Moniz, J.M.N. (2003). A enfermagem e a pessoa idosa: a prática de cuidados como experiência formativa Loures. Loures: Lusociência.

Moraes, E., Moraes F., \& Lima, S. (2010). Características biológicas e psicológicas do envelhecimento. Revista Médica de Minas Gerais, 20(1), 67-73. 
Nakatano, A., Souto, C., Paulette, L., Melo, T., \& Sousa, M. (2003). Perfil dos cuidadores informais de idosos com déficit de autocuidado atendidos elo Programa de Saúde da Família. Revista Eletrônica de Enfermagem, 5(1), 15-20.

Neri, A.L. (2001). Desenvolvimento e envelhecimento: perspectivas biológicas, psicológicas e sociológicas. Campinas: Papirus.

OMS (1993). Clasificación Internacional de las Enfermedades (CIE). Trastornos mentales $y$ del comportamiento - Criterios diagnósticos de investigación. Madrid: Meditor.

Pearlin, L. I., Mullan, J. T., Semple, S. J., \& Skaff, M. M. (1990). Caregiving and the stress process: an overview of concepts and their measures. The Gerontologist, 30(5), 583-594.

Ramos, B. (2004). Culture, Ethnicity, and Caregiver Stress among Puerto Ricans.
Journal of Applied Gerontology, 23(4), 469-486.doi: 10.1177/0733464804271281

Salgado, M. A. (1982). O significado da velhice no Brasil. São Paulo: SESC.

Salthouse, T. A. (1996). The Processing-Speed Theory of Adult Age Differences in Cognition. Psychological Review, 103(3), 403-428.

Sequeira, C. (2010). Cuidar de idosos com dependência física e mental. Lisboa: Lidel.

Sousa, L., Figueiredo, D., \& Cerqueira, M. (2004). Envelhecer em família. Porto: Âmbar.

Vitaliano, P., Scanlan, J., \& Zhang, J. (2003). Is caregiving hazardous to one's physical health? A meta-analysis. Psychological Bulletin, 129(6), 946-72.

Weineck, J. (2001). Biologia do esporte. Barueri: Manole. 